\title{
CORRECTION
}

View Article Online

View Journal I View Issue

\section{Correction: Tunable self-assembly of Irinotecan- fatty acid prodrugs with increased cytotoxicity to cancer cells}

Cite this: J. Mater. Chem. B, 2016 4,3842

Chunqiu Zhang, ${ }^{a}$ Shubin Jin, ${ }^{a}$ Xiangdong Xue, ${ }^{a}$ Tingbin Zhang, ${ }^{a}$ Yonggang Jiang, ${ }^{a}$ Paul C. Wang*bc and Xing-Jie Liang*a

DOI: $10.1039 / c 6 t b 90070 d$

Correction for 'Tunable self-assembly of Irinotecan-fatty acid prodrugs with increased cytotoxicity to www.rsc.org/MaterialsB cancer cells' by Chunqiu Zhang et al., J. Mater. Chem. B, 2016, DOI: 10.1039/c6tb00612d.

The authors wish to clarify that in the Experimental section, Preparation of Irinotecan-fatty acid prodrug nanoparticles (NPs), the amount of each prodrug dissolved in DMSO should be $0.6 \mathrm{mg}$, and not $6.0 \mathrm{mg}$ as stated in the published article.

The Royal Society of Chemistry apologises for these errors and any consequent inconvenience to authors and readers.

\footnotetext{
${ }^{a}$ Chinese Academy of Sciences (CAS) Center for Excellence in Nanoscience and CAS Key Laboratory for Biological Effects of Nanomaterials \& Nanosafety, National Center for Nanoscience and Technology, No. 11 Beiyitiao, Zhongguancun, Beijing 100190, China. E-mail: liangxj@nanoctr.cn

${ }^{b}$ Fu Jen Catholic University, Taipei, 24205, Taiwan

${ }^{c}$ Laboratory of Molecular Imaging, Department of Radiology, Howard University, Washington, D.C. 20060, USA. E-mail: pwang@Howard.edu
} 